\title{
Environmental influences on the pace of brain development
}

\section{Ursula A. Tooley (1), Danielle S. Bassett (D) and Allyson P. Mackey (D)}

Abstract | Childhood socio-economic status (SES), a measure of the availability of material and social resources, is one of the strongest predictors of lifelong well-being. Here we review evidence that experiences associated with childhood SES affect not only the outcome but also the pace of brain development. We argue that higher childhood SES is associated with protracted structural brain development and a prolonged trajectory of functional network segregation, ultimately leading to more efficient cortical networks in adulthood. We hypothesize that greater exposure to chronic stress accelerates brain maturation, whereas greater access to novel positive experiences decelerates maturation. We discuss the impact of variation in the pace of brain development on plasticity and learning. We provide a generative theoretical framework to catalyse future basic science and translational research on environmental influences on brain development.

Children's early experiences are associated with important later-life outcomes, including their earnings $s^{1}$, educational attainment ${ }^{2}$, physical well-being ${ }^{3}$ and mental health ${ }^{4}$. How are children's experiences embedded in their developing brains to broaden, or constrain, their opportunities to live happy and healthy lives? Much of what we know about links between early experiences and adult outcomes has come from research on socio-economic status (SES). A multidimensional construct, SES is typically measured at the household level (for example, parental income, education or occupation) or the neighbourhood level (for instance, neighbourhood crime rate, poverty levels or median income). Higher SES is associated with lower exposure to stress, and with greater access to cognitive enrichment, such as high-quality education, child-directed language, books and toys. Variation in childhood SES has been associated with variation in measures of brain structure and function ${ }^{5-8}$. However, surprisingly little is known about whether and how experiences associated with childhood SES affect the trajectory of brain maturation.

Here, we synthesize evidence that experiences associated with childhood SES affect not only the outcome, but also the pace of brain development, and consider the implications of early brain development for plasticity in childhood. We focus on whole-brain cortical measures of structure and function because, as a broad and multidimensional construct, SES probably exerts effects on a complex constellation of brain regions and their connections. We highlight the few longitudinal studies on SES and brain development but, because these studies are rare, we also draw on cross-sectional studies of relationships between SES and brain structure and function across development ${ }^{9}$. We consider how experiences, including stress, cognitive enrichment and environmental variability, influence brain maturation and plasticity. We close by outlining promising future directions for research on how children's early experiences lead to disparities in later-life outcomes.

\section{Structural brain development Cortical thickness. Cortical thickness} increases in the prenatal and immediate postnatal period, driven by dendritic and axonal growth as well as synaptogenesis ${ }^{10}$. Peak synaptic density and peak cortical thickness are reached at different times across the brain, with sensory regions showing faster development and earlier peaks, and association regions showing slower developmental trajectories ${ }^{11,12}$ (FIG. 1). The cortex thickens before 2 years of age, before undergoing widespread thinning across a protracted period starting between 2 and 5 years of age, and continuing through adolescence and early adulthood. Thinning is attributed to both regressive (synaptic pruning) and progressive (myelination) processes ${ }^{13,14}$. In adulthood, a thicker cortex is associated with larger, more complex pyramidal neurons ${ }^{15}$. Cortical surface area increases during childhood and into early adolescence, with the greatest increases occurring first in sensory areas, and latest in association areas ${ }^{16,17}$.

Children and adolescents from higher-SES environments generally have thicker cortex than those from lower-SES environments ${ }^{8,18-20}$, but there is evidence that relationships between SES and cortical thickness vary with age (FIG. 1). In the first postnatal year, when the cortex rapidly thickens, higher paternal education is associated with thinner cortex, particularly in the frontal lobes ${ }^{21}$. This pattern is suggestive of more prolonged maturational processes in infants from higher-SES backgrounds. Later in development, in youth aged 3-20 years, SES moderates the negative relationship between age and cortical thickness such that youth from lower-SES backgrounds show a steeper curvilinear decrease in cortical thickness at a younger age than do youth from higher-SES backgrounds ${ }^{22,23}$. Adolescents aged 12-18 years in low-income households show a steeper curvilinear relationship between age and cortical thickness than do adolescents in high-income household ${ }^{24}$. For females, but not males, in low-income households, living in high-inequality neighbourhoods is again associated with a steeper negative relationship between age and cortical thickness ${ }^{24}$. This evidence is consistent with the hypothesis that lower SES is associated with accelerated cortical thinning throughout childhood and adolescence. However, not all findings align with this hypothesis. Two recent studies examined youth aged 5-25 years ${ }^{25}$ and 14-19 years $^{26}$ and did not find that SES moderated relationships between age and cortical thickness, although the former study reported positive correlations between SES 

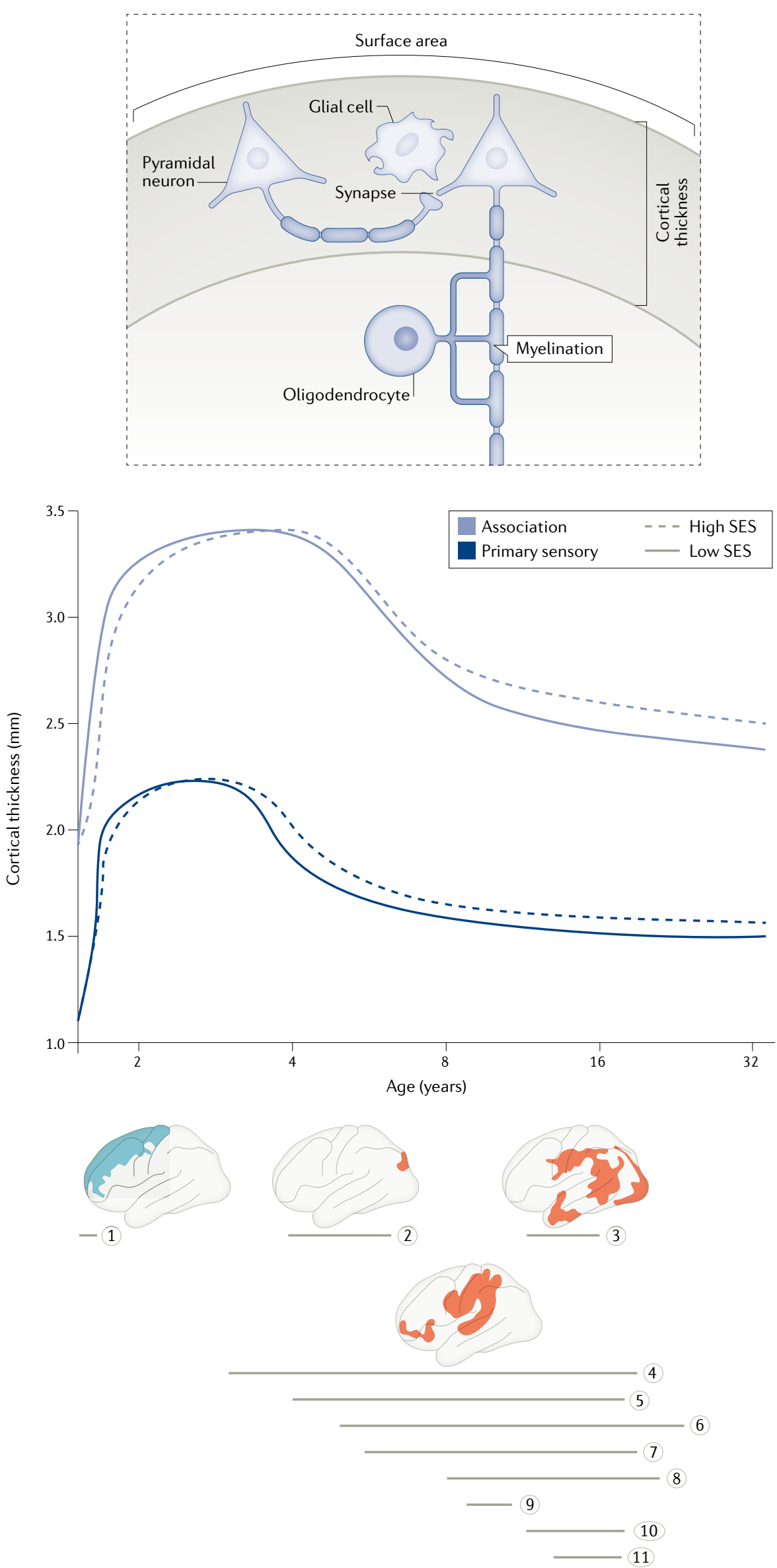

Fig. 1 | Associations between socio-economic status and cortical thickness. Trajectories shown in light and dark blue are conceptual, based on findings interpolated across multiple studies. Horizontal grey lines represent the age ranges of individual studies, as shown on the horizontal axis. Brain regions shown in blue indicate negative relationships between socio-economic status (SES) and cortical thickness (REF. ${ }^{21}$ corresponds to grey line 1). Brain regions shown in red indicate positive relationships between SES and cortical thickness (grey line 2, REF. ${ }^{19}$; grey line 3, $\mathrm{REF}^{8}$; grey line 4, REF. ${ }^{22}$; grey line 5, REF. ${ }^{18}$; grey line 6, REF. ${ }^{25}$; grey line 7, REF. ${ }^{107}$; grey line 8, REF. ${ }^{36}$; grey line 9, REF. ${ }^{20}$; grey line 10, REF. ${ }^{24}$; grey line 11 , REF. $\left.{ }^{26}\right)$. These curves are consistent with more modest main effects of SES on cortical thickness when averaging is done across large age ranges than when small age ranges are focused upon. The inset shows a schematic of potential cellular underpinnings of cortical thickness as measured by MRI: glial number and size, neuron number and size, synaptic complexity and myelination ${ }^{14-16}$. Cells are enlarged relative to cortical thickness to show detail. Brain image corresponding to grey line 1 adapted with permission from REF. ${ }^{21}$, OUP. Brain image corresponding to grey line 2 adapted with permission from REF. ${ }^{19}$, CC BY 4.0 (https:// creativecommons.org/licenses/by/4.0/). Brain image corresponding to grey line 3 adapted with permission from REF. ${ }^{8}$, Sage Publishing. Brain image corresponding to grey line 4 adapted with permission from REF. ${ }^{22}$, CC BY 4.0 (https:// creativecommons.org/licenses/by/4.0/).

and cortical thickness. However, examining a large age range such as 5-25 years might obscure interaction effects that vary over the course of development, and SES-related variability in the rate of cortical thinning during late adolescence when thinning has slowed may be minimal (FIG. 1). In addition, neither study examined non-linear relationships between age and cortical thickness moderated by SES.

Surface area. Fewer studies have examined associations between SES and cortical surface area development. In infancy, surface area is not related to parental education or income ${ }^{21}$. In late childhood and adolescence, however, higher SES is associated with greater surface area ${ }^{25-27}$. In an analysis of the Pediatric Imaging, Neurocognition, and Genetics (PING) dataset, researchers applied sample weights to structural brain imaging data collected from children aged 3-18 years to create a 'weighted sample' approximating the distribution of SES, race/ethnicity and sex in the US population. When the researchers used the weighted sample to examine associations between surface area and age, the surface area peak shifted earlier as compared with the unweighted sample, 
consistent with an interpretation of earlier or faster brain maturation in children from lower-SES backgrounds, who were under-represented in the original sample ${ }^{28}$. In a recent longitudinal study of adolescents, higher SES was associated with a smaller decline in total surface area between 14 and 19 years of age ${ }^{26}$.

Cellular underpinnings. The cellular processes that underlie cortical thickness and surface area measures obtained with MRI are still under active investigation. As noted already, cortical thickness is positively associated with synaptic density, and is negatively associated with myelination ${ }^{14,15}$. One possibility is that experiences associated with low SES drive earlier curtailment of synaptic proliferation and a subsequently decreased range for optimal synaptic pruning and wiring of functional networks. Computational models of synaptic proliferation suggest that synaptic overgrowth and then pruning of weak synapses maximizes network performance, given the metabolic constraints of the brain ${ }^{29}$. In biologically motivated models of network development, delaying synaptogenesis in higher-order layers of a network leads to greater energy efficiency and faster learning after development ${ }^{30}$. Moreover, networks with more initial connections are better able to learn than networks with fewer initial connections $^{31}$. Computational models of synaptic proliferation and subsequent pruning early in development have identified a trade-off between rapid development, which enables earlier independence and less parental input, and optimal adult neural performance $^{32}$. SES-associated differences in early synaptic proliferation would affect the development of functional connectivity, which we examine in the following section.

\section{Functional network development}

A key goal of brain development is to establish efficient, specialized cortical systems. Functional activation of specific systems can be studied by imaging individuals performing well-designed tasks, but SES-associated differences in task accuracy and the interpretation of stimuli can affect conclusions about the underlying anatomy ${ }^{33}$. By contrast, data collected when participants relax inside the scanner - that is, resting-state functional MRI (rs-fMRI) data - can be used to study all systems simultaneously without task confounds ${ }^{34}$. Components of a functional system show statistically similar patterns of fluctuations in blood oxygenation, commonly referred to as functional connectivity ${ }^{35}$.
Resting-state analyses have generated conflicting answers to the question of whether higher SES is associated with faster functional maturation. One compelling study integrated grey and white matter structure with regional rs-fMRI measures to develop a model to classify individuals' ages. It was found that individuals aged 8-22 years from lower-SES backgrounds were more likely to be classified as adults than their higher-SES counterparts ${ }^{36}$. Other rs-fMRI studies also suggest that lower SES is associated with faster functional development: in youth aged 6-17 years, lower SES was associated with weaker connectivity in corticostriatal connections that typically showed decreases in strength with age over development ${ }^{37,38}$. However, some studies have found the opposite pattern: higher SES has been associated with greater functional connectivity between limbic regions that typically show age-related increases in functional connectivity over development ${ }^{39-41}$. These studies largely examined patterns of regional metrics or connectivity between specific sets of regions rather than testing for broad effects of SES on the pace of network development throughout the brain. However, region-to-region connectivity can be strengthened by repeated co-activation, just as cells that fire together will wire together. Therefore, it is difficult to infer broad developmental processes from examining links between specific regions ${ }^{42}$.

Newer approaches to analysing rs-fMRI data are computationally better suited to test the hypothesis that higher childhood SES is associated with protracted development of functional networks across the entire cortex. A network science approach, in particular, represents the brain as a collection of nodes (regions) and edges (connections), enabling us to address the whole-brain pattern of connectivity ${ }^{43,44}$. The resulting network architecture can then be quantitatively characterized with use of tools from graph theory to identify key properties relevant to maturation ${ }^{45}$. Two such properties are segregation and integration, both of which change during development ${ }^{46}$. Segregation quantifies the presence of groups or subnetworks of densely interconnected nodes in a network, whereas integration assesses the extent to which information can be rapidly combined from distributed regions ${ }^{43}$. Integration has a distinct meaning when one is interpreting diffusion data compared with when one is interpreting functional data ${ }^{47}$ (BOX 1). Together, integration and segregation constitute the unique property of small-worldness found in adult brain networks: the perhaps counterintuitive presence of high levels of both segregation and integration at many different scales (see REF. ${ }^{48}$ for a recent review). Given the associations between functional network segregation at rest and cognitive abilities ${ }^{35,49}$, and that most research on SES and functional network development has examined segregation rather than integration, we focus specifically here on measures of functional network segregation.

Segregation in brain networks changes markedly over development, and can be measured at several scales. One measure of segregation at the nodal level is the clustering coefficient, which quantifies the connectivity in a node's immediate neighbourhood. At the mesoscale and global levels, modularity captures the extent to which a network can be divided into distinct subnetworks or modules, and system segregation captures the extent to which systems within a functional network are distinctly partitioned ${ }^{35}$. A coarse proxy for system segregation is within-system connectivity.

Studies of prenatal development show that a segregated network structure is present even in utero, with modular subnetworks that coarsely resemble those found in adults ${ }^{50,51}$. Inter-regional variation in the width of time windows of synaptogenesis during prenatal and early postnatal development (for example, as seen in REF. ${ }^{11}$ ) gives rise to the highly connected hub nodes and modular structure seen in adult brain networks ${ }^{52,53}$. Similarly to structural brain development ${ }^{11,12}$, functional subnetworks underlying sensory systems become established at an earlier age than do the subnetworks underlying association systems $^{54,55}$. Mesoscale segregation increases with age later in childhood and adolescence, probably reflecting the refinement of network architecture; higher-order association systems in particular become more segregated with development ${ }^{49,56}$ (although some studies do not find positive associations between age and segregation during adolescence, perhaps owing to differences in age range and node or edge definitions; see REF. ${ }^{57}$ ). Maturation at the cellular level probably gives rise to these macroscale developmental changes. Inhibitory interneurons have a role in limiting resting-state functional connectivity and establishing the boundaries between brain regions that are necessary for network segregation $^{58}$. In addition, connection strength is associated with microscale properties of connected brain regions, including the size and complexity of layer III pyramidal neurons ${ }^{59,60}$, cytoarchitectonic similarity ${ }^{61}$ and excitatory-inhibitory receptor balance ${ }^{62}$. 
Only a few studies have examined associations between SES and functional brain development using a network science approach (FIG. 2), and these studies have used different measures of segregation. Although the use of different measures of segregation at different scales makes an overarching pattern difficult to interpret, here we draw upon existing studies to sketch a theoretical model for future work to detail. One study ${ }^{63}$ of infants less than 1-year-old found marginally significant associations between higher SES and both similarity to adult systems and within-system connectivity, a proxy for system segregation. The study's authors interpret these observations as indicative that greater maturation is associated with higher SES. However, the significant associations were found only at 6 months of age and not at the other time points examined $(1,3,9$ or 12 months). In another study, youth aged 8-22 years from high-SES neighbourhoods show a stronger association between age and local segregation - clustering - than did youth from low-SES neighbourhoods ${ }^{64}$. Although the study authors also examined a mesoscale measure of segregation, namely modularity, the moderating effect of SES on associations between age and modularity was accounted for by local segregation, suggesting that the fundamental driver was variation in local network topology. Specifically, during late childhood, youth from high-SES neighbourhoods showed lower local cortical functional segregation than did youth from low-SES neighbourhoods. However, youth from high-SES neighbourhoods showed a steeper positive relationship between segregation and age during adolescence, such that by their early 20s, they showed greater functional network segregation than youth from low-SES neighbourhoods. Another study of individuals in a similar age range (6-17 years) revealed an interaction between household and neighbourhood SES, such that among youth in low-SES neighbourhoods, higher household SES is associated with greater local functional network segregation

Box 1 | Environmental effects on white matter development

If lower socio-economic status (SES) is associated with accelerated brain maturation, we would expect to see differences in the pace of brain maturation reflected in diffusion-based measures of white matter; however, few studies have examined this topic. Typically, studies examining white matter tend to consider fractional anisotropy (FA): the degree of restricted diffusion in a principal direction $\left(\lambda_{1}\right)$ compared with orthogonal directions $\left(\lambda_{2}\right.$ and $\lambda_{3}$; see the figure). FA is generally interpreted as a measure of the integrity of a white matter fibre tract. Streamline count is a measure of how many 'fibres' can be reconstructed between two brain regions ${ }^{213}$. Structural brain networks can be constructed from measures of

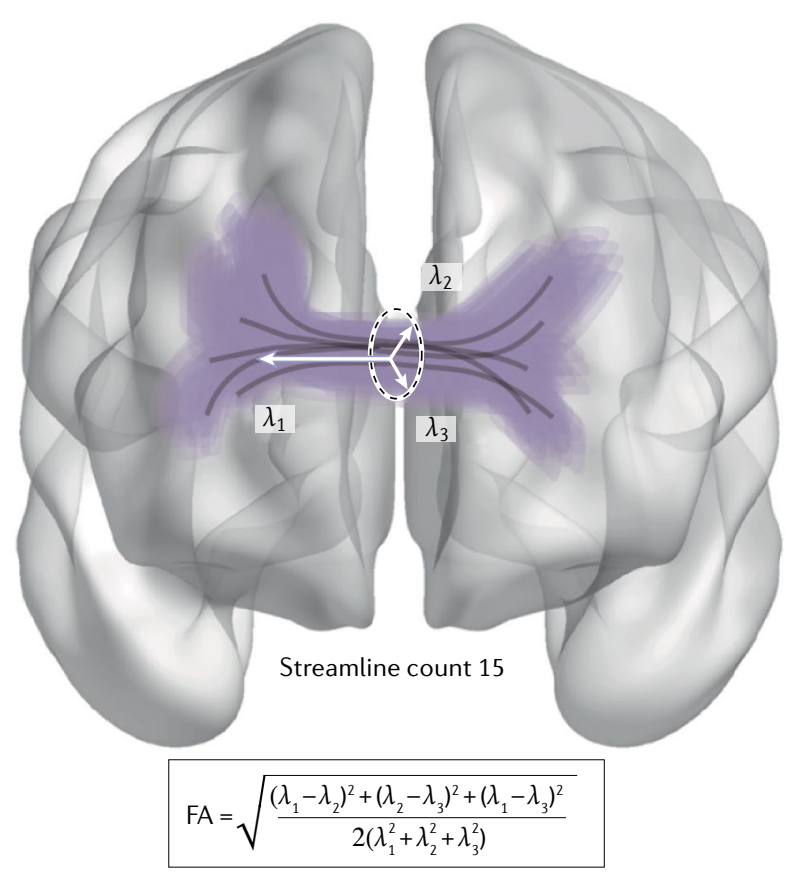

regional streamline count or diffusion scalar values averaged along a tract, such as FA.

FA increases steeply in the first few years of life and then more slowly throughout childhood and adolescence ${ }^{214,215}$. In developmental studies, group differences in white matter integrity between children from high-SES backgrounds and children from low-SES backgrounds have been identified across various ages, consistently showing that higher SES is associated with higher FA in early childhood (4-7 years) ${ }^{216}$, in middle childhood (8-10 years) ${ }^{217}$, through adolescence (6-19 years and 17-23 years) ${ }^{107,218}$ and into young adulthood (18-27 years) ${ }^{219}$. Children from higher-SES environments show higher global efficiency of their structural brain networks, indicating that their white matter has many short paths between regions, suggestive of relatively greater integration than in networks of children from lower-SES backgrounds ${ }^{220}$. However, none of these developmental studies examined age-SES interactions. Importantly, measures of FA are related to both axon coherence (compact bundling of several axons in a similar orientation) and myelination, and may also conflate experience-expectant (age-related) myelination with experience-dependent myelination, impairing our ability to detect environmental influences on the rate of maturation. (assessed by the clustering coefficient) in the prefrontal cortex ${ }^{65}$. The available evidence is consistent with the hypothesis that higher SES is associated with more protracted functional network development, with youth from high-SES backgrounds showing more widespread connectivity and thus lower segregation early in development, before the rapid development of a more segregated network architecture that continues into adulthood $^{10,11}$.

In sum, these studies suggest that the effects of SES on structural development may be reflected in functional development, such that the extended period of structural development associated with high SES gives rise to a longer, slower trajectory of functional network segregation during development, leading to greater segregation. Although longitudinal studies with consistent measures of functional network organization necessary to strictly test these hypotheses do not yet exist, we draw upon existing work to sketch a theoretical model for future work. Lower SES is associated with faster thinning and blunted functional remodelling during childhood and adolescence. In late adolescence and young adulthood, individuals from higher-SES backgrounds show greater cortical thickness and greater segregation than do individuals from lower-SES backgrounds, perhaps as a result of differences in maturation rate. The findings described above also suggest that associations between SES and functional network segregation might follow a progression from local to global across the lifespan, with associations in childhood and early adolescence evident at the local level, and associations at the mesoscale and global level visible later in life. However, more work is needed to understand whether there are truly differing associations at different scales, as few studies thus far have examined multiple measures of segregation in conjunction.

We now turn to two of the most well-studied putative mechanisms underlying SES-associated differences in brain development: stress and cognitive enrichment ${ }^{5,66}$. Previous conceptual models have organized variation in early experiences along dimensions of threat (similar to stress) and deprivation (the opposite of cognitive enrichment) ${ }^{67-69}$. We review these factors as possible contributors to the effects of SES on the pace of brain development.

\section{Stress}

Lower SES is consistently associated with greater chronic stress ${ }^{70}$, and prior work extensively reviewed the links between SES and multiple conceptualizations 


\section{PERSPECTIVES}

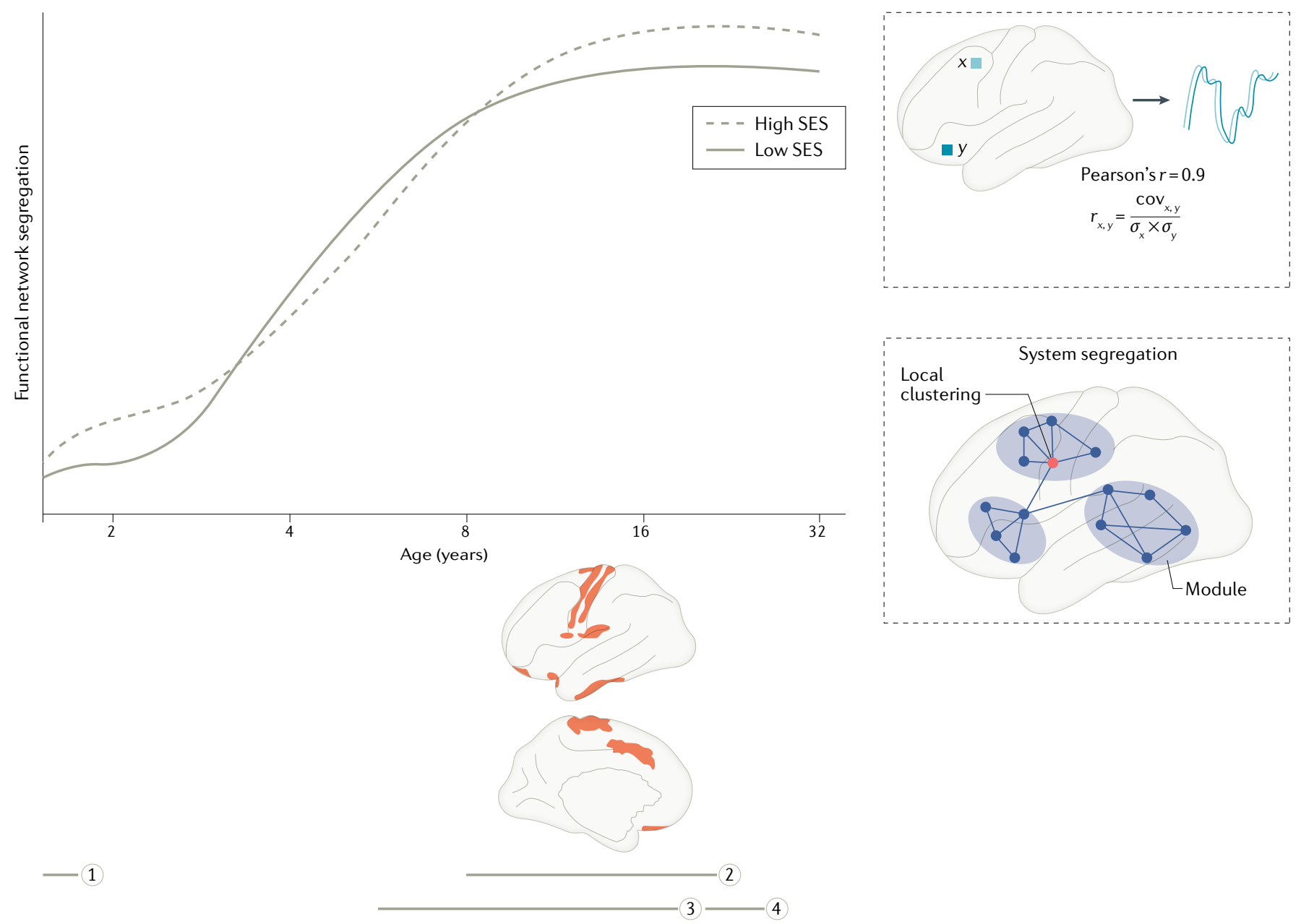

Fig. 2 | Associations between socio-economic status and functional brain network segregation. Trajectories shown in solid and dashed grey lines are conceptual, based on findings interpolated across multiple studies. Horizontal grey lines represent the age ranges of individual studies, as shown on the horizontal axis (grey line 1, REF. ${ }^{63}$; grey line 2, REF. ${ }^{64}$; grey line 3; REF. ${ }^{65}$; grey line 4, REF. ${ }^{196}$ ). Brain regions shown in red indicate socio-economic status (SES)-associated differences in functional network segregation, with adolescents from higher-SES backgrounds showing stronger positive associations between age and segregation. Curves are drawn to be consistent with functional network segregation across the studies shown; the studies used a range of measures of segregation, as illustrated in the bottom-right inset. The top-right inset illustrates a common metric of functional connectivity used to estimate functional brain networks: the Pearson product-moment correlation coefficient. Brain images in the lower part of the figure adapted with permission from REF. ${ }^{64}$, OUP. of stress ${ }^{68,71-75}$. There are at least three mechanisms by which chronic stress exposure could accelerate brain development. The first is that repeated use of stress-detection and stress-regulation circuitry, including the amygdala and medial prefrontal cortex, could lead to faster maturation of that circuitry ${ }^{76,77}$. The second is that stress could cause faster ageing of the entire body by increasing glucocorticoid levels and allostatic load (physiological wear and tear) and by promoting activation of inflammatory processes ${ }^{78}$. These same physiological processes can be activated by other experiences associated with lower SES, including exposure to environmental toxins (such as lead or air pollution) ${ }^{79}$, poorer sleep quality ${ }^{80}$ and less opportunity for physical activity ${ }^{81-84}$. Stress is associated with accelerated cellular ageing, marked by changes in epigenetic processes such as methylation ${ }^{85,86}$, which are detectable in childhood $^{87,88}$. Individuals from lower-SES backgrounds tend to enter puberty earlier, and this effect is driven most strongly by experiences of threat ${ }^{89-92}$. Earlier puberty in turn might also accelerate brain maturation. One study found that the expression of the genes encoding the glucocorticoid receptor and the androgen receptor explained the most variance in cortical thinning in low-income female adolescents living in high-inequality neighbourhoods, suggestive of links between stress and both accelerated puberty and cortical thinning ${ }^{24}$. A third possible mechanism by which chronic stress may accelerate brain development is that young individuals process threat as an overall signal of lack of protection and support - that is, they receive cues that the environment requires maturity - and this triggers adaptive top-down processes that cause development to proceed more quickly. This was recently termed the 'developmental support hypothesis' (see REF. ${ }^{93}$ ), and aligns with much evolutionary life-history research, including cross-species findings that parental investment is associated with slower maturation ${ }^{93-95}$. Understanding which, if any, of these mechanisms affect the pace of brain development is essential for determining when and how it might be possible to intervene.

Animal models of early-life stress allow us to address issues of causality that cannot be examined in humans. The animal paradigm most analogous to the economic 
deprivation and stress associated with SES is the limited bedding and nesting model in rodents, which involves limiting the dam's access to sufficient bedding and nesting material. Although the limited bedding paradigm fails to capture many of the social, emotional and cognitive aspects of being raised in a low-SES environment, this constraint does result in fragmented and unpredictable nurturing behaviours and increased glucocorticoid release in the pups $^{96,97}$. Offspring of the dams exposed to this paradigm show earlier declines in the levels of markers of postnatal neurogenesis in the hippocampus, earlier increases in the levels of markers of synaptic maturity, earlier increases in the level of myelin basic protein and impairments in cognitive function ${ }^{98-100}$. They also show an initial increase in neuronal proliferation in the hippocampus in early life, but at later times show reduced numbers of neurons and reduced hippocampal volume, suggestive of an earlier peak in neurogenesis ${ }^{101}$. Prefrontal areas and the hippocampus show reduced spine density following exposure to this paradigm in the early postnatal period. These changes are associated with impairments in cognitive function ${ }^{102,103}$ that are prevented by blocking the effect of corticotropin-releasing hormone, a stress-linked neuropeptide, immediately following exposure to this paradigm. This finding is consistent with a large body of work showing that some effects of the early environment are modulated by glucocorticoids ${ }^{85,104}$. We now turn to the question of whether SES differences in cognitive enrichment or deprivation also drive differences in the pace of brain development.

\section{Cognitive enrichment}

Exposure to a complex environment with a variety of experiences and diverse learning materials is known as cognitive enrichment. The absence of cognitive enrichment is considered deprivation ${ }^{67,68}$. Children growing up in higher-SES homes tend to be exposed to more complex and cognitively stimulating environments ${ }^{105}$, and cognitive enrichment is associated with improved cognition in youth independent of stress exposures $^{69,106-108}$. In one study, cognitive stimulation also mediated associations between SES and cortical thickness in prefrontal areas ${ }^{107}$, highlighting its potential role as a mechanism of the influence of SES on brain development in childhood. Recapitulating these findings, SES-associated differences in children's cognitive function have been reported to be mediated by cognitive enrichment in the home ${ }^{109}$.

\section{Box 2 | Cellular and molecular mechanisms of plasticity}

In animal models, the study of critical or sensitive periods, windows of heightened plasticity when brain development depends on specific expected environmental inputs, has yielded insight into the mechanisms of the regulation of plasticity, summarized in the table along with neuroimaging measures well suited to track these mechanisms ${ }^{123,221}$. Excitatory-inhibitory circuit balance, driven by the maturation of parvalbumin-positive $\left(\mathrm{PV}^{+}\right)$inhibitory interneurons, leads to periods of heightened plasticity, and molecular 'brake'-like regulators limit plasticity later in development ${ }^{222}$. Accumulation of regulators such as the homeobox protein OTX2 and brain-derived neurotrophic factor (BDNF) trigger the maturation of $\mathrm{PV}^{+}$neurons and opening of periods of heightened plasticity ${ }^{124}$. Subsequently, brake-like factors such as perineuronal nets and myelin maintain the closure of periods of heightened plasticity, stabilizing neural circuitry to limit rewiring during adulthood. In humans, these brake-like factors accumulate during development in parallel with the progression of structural changes such as cortical thinning, first in primary sensory and motor areas and later in higher-order association areas ${ }^{223-227}$. Neuromodulators such as dopamine, acetylcholine and serotonin can upregulate plasticity even once structural brakes are in place $\mathrm{e}^{143,222,228}$.

\begin{tabular}{ll} 
Cellular or molecular measure & Neuroimaging measure \\
Excitation-inhibition balance & $\begin{array}{l}\text { Magnetic resonance spectroscopy, glutamate } \\
\text { chemical exchange saturation transfer, } \\
\text { GABA chemical exchange saturation transfer }\end{array}$ \\
$\begin{array}{l}\text { Extracellular matrix organization } \\
\text { (including perineuronal nets) }\end{array}$ & $\begin{array}{l}\text { Multicompartment diffusion imaging (for example, } \\
\text { neurite orientation dispersion and density imaging } \\
\text { or soma and neurite density imaging) }\end{array}$ \\
\hline Myelin & $\begin{array}{l}\text { Fractional anisotropy or mean diffusivity from } \\
\text { diffusion imaging; multicompartment diffusion } \\
\text { imaging; T1-weighted to T2-weighted ratio; } \\
\text { magnetization transfer; quantitative MRI }\end{array}$ \\
\hline $\begin{array}{l}\text { Levels of neurotransmitters (such as } \\
\text { dopamine, acetylcholine or serotonin) }\end{array}$ & $\begin{array}{l}\text { Positron emission tomography, functional MRI or } \\
\text { resting-state functional MRI of neuromodulatory } \\
\text { nuclei }\end{array}$ \\
\hline
\end{tabular}

Some models suggest that the absence of cognitive enrichment in specific domains leads to accelerated synaptic pruning in brain regions that process complex cognitive and social stimuli ${ }^{67,68}$. The converse of this argument is that specific cognitive inputs might delay synaptic pruning in relevant brain circuitry.

As in studies of stress, animal models allow us to investigate the causal influence of cognitive enrichment on brain development. Environmental enrichment paradigms typically have two main components: novel objects and novel social partners. Environmental enrichment in both juvenile and adult animals has been shown to lead to increased cortical thickness ${ }^{110,111}$, driven by increases in dendritic volume and branching ${ }^{112,113}$, dendritic spine count ${ }^{112,114}$, synaptogenesis and glial proliferation ${ }^{115,116}$ (reviewed in REF. ${ }^{117}$ ). As little as 4 days of enrichment produces measurable changes in cortical thickness in rodents ${ }^{118,119}$, and longer exposure is associated with longer retention of increased thickness after return to a standard environment ${ }^{120}$. Enrichment may also affect cortical surface area, but it is not commonly measured ${ }^{121}$. Increased synaptogenesis, glial proliferation and dendritic plasticity could indicate a prolonged period of maturation leading to more complex brain circuitry, as computational models that suggest early synaptic overgrowth and overall slower development are advantageous for adult network abilities ${ }^{32,122}$. In sum, there is some evidence that children's early experiences of stress and cognitive enrichment influence the pace of brain development.

\section{Consequences for plasticity}

Understanding how children's experiences affect the pace of brain maturation has consequences for understanding brain plasticity. Brain plasticity can be conceptualized in two ways: as a process and as a potential. The process of brain plasticity, including long-term potentiation and other structural and functional changes in response to experience, occurs throughout life. However, the brain's plasticity as potential for change varies with age. Developmental processes, including myelination, inhibition and the formation of perineuronal nets (PNNs; lattice-like extracellular structures that enwrap neurons and act as a physical brake on plasticity) decrease the brain's ability to change as children get older ${ }^{123,124}$ (BOX 2). If brain development proceeds more quickly in children from low-SES backgrounds, windows of high plasticity could also close 
more quickly in these children, reducing the brain's sensitivity to future experiences. In this section, we review evidence from animal models that experiences of stress and cognitive enrichment affect plasticity. Most of this research was done in adult animals, but the results suggest that these experiences would affect plasticity during development as well.

Studies in animal models have broadly shown that early-life stress decreases synaptic plasticity and promotes the developmental processes that reduce plasticity (such as inhibition and myelination). Offspring of dams exposed to the limited-bedding paradigm show earlier increases in the levels of markers of synaptic maturity, earlier increases in the level of myelin basic protein, an increased number of PNNs and reductions in adult synaptic plasticity, accompanied by impairments in cognitive function, compared with control offspring ${ }^{98,99,101,125}$. The limited-bedding paradigm also causes reduced spine plasticity in the offspring's prefrontal cortex and hippocampus ${ }^{102,103}$. Increased myelination is not always observed following early-life stress: one study found that early social isolation leads to a decrease in myelination in the prefrontal cortex ${ }^{126}$. Therefore, the impact of stress on myelination may depend on the type of stressor and the brain area examined. There are also indirect links between early-life stress and plasticity: early-life stress accelerates pubertal timing (age of onset of pubertal development or age at menarche), and ovarian hormones increase cortical inhibition ${ }^{92,127,128}$. Studies of stress on plasticity in humans are rare. One study of post-mortem brains found that individuals who were exposed to child abuse had increased numbers of mature myelinating oligodendrocytes in the ventral medial prefrontal cortex ${ }^{129}$. Another study used neuroimaging to show that veterans with post-traumatic stress disorder had higher T1-weighted/T2-weighted MRI signal, a marker of myelination, in the hippocampus ${ }^{130}$. Both studies are consistent with the hypothesis that stress increases myelination, and may thereby limit plasticity.

Environmental-enrichment paradigms prolong and enhance plasticity. Enrichment during the juvenile period decreases the number of $\mathrm{PNNs}^{131}$, enhances synaptic plasticity in the form of long-term potentiation and depression ${ }^{132}$ and influences parvalbumin-positive neuron expression ${ }^{131,133,134}$. In adulthood, enrichment keeps inhibition at juvenile levels, prolonging early periods of plasticity ${ }^{135-137}$.

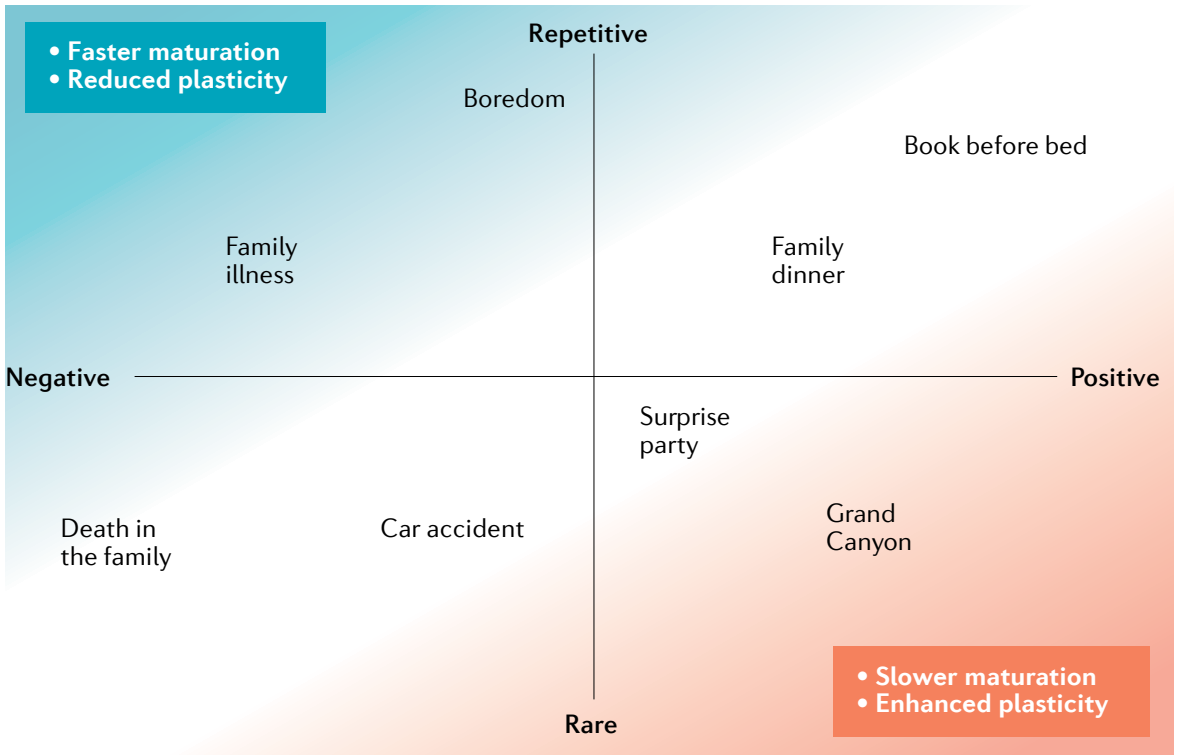

Fig. 3 | Integrative theory: childhood experiences affect the pace of brain development. According to our model, experiences that are chronic or repetitive and negative encourage faster maturation and increase allostatic load, potentially restricting plasticity. Experiences that are rare and positive, triggering surprise and awe, are associated with strong neurochemical signals to delay maturational processes and enhance plasticity. Experiences in the other quadrants (rare and negative, or repetitive and positive) are predicted to have smaller effects on the global pace of maturation.

Enrichment paradigms can also enhance plasticity in adults long past juvenile critical periods ${ }^{138}$ by reducing inhibition ${ }^{137,139}$, decreasing PNN stability ${ }^{139-141}$ or increasing myelin remodelling ${ }^{142}$, all potent contributors to plasticity. Environmental enrichment increases neuronal secretion of the cytokine interleukin-33 (IL-33), which signals to microglia to engulf PNNs, increasing synaptic plasticity ${ }^{141}$. Environmental enrichment also enhances levels of neurotransmitters, including noradrenaline, dopamine and serotonin, which increase cortical plasticity and facilitate cortical remodelling ${ }^{143-146}$. Mice lacking the dopamine D2 receptor or the dopamine D4 receptor fail to benefit in longevity from enriched environments $^{147,148}$. The social interaction component of an enriched environment increases release of oxytocin, which enhances plasticity $^{149}$ and protects against stress-related changes in plasticity ${ }^{150,151}$. To our knowledge, studies examining the impact of cognitive enrichment on plasticity in humans do not yet exist.

Stress and cognitive enrichment broadly capture the valence of experiences: stressful experiences are negative and should be avoided, whereas environmental enrichment paradigms are designed to be positive and rewarding. However, valence is not the only salient property of such experiences. The timing of experiences also has implications for plasticity (FIG. 3). Repeated exposure to the same experience should signal that the experience is more likely to occur consistently in the future, and that the brain should optimize to respond to it, even at a cost to plasticity. Experience-dependent myelination and PNN formation are two potential mechanisms by which repeated activation of brain circuitry might lead to reduced plasticity ${ }^{124}$.

Empirical evidence in humans supports the theory that rote practice accelerates maturation of specific brain circuits. In adults, after several weeks of repetitive task practice, functional systems involved in the task become more segregated from each other ${ }^{152-154}$, mimicking network segregation during development ${ }^{49,56}$. Similarly, working memory training in young children aged 4-6 years results in changes in attentionrelated brain activity that resemble those that occur with maturation ${ }^{155}$. Thus, some brain systems may mature more quickly in high-SES environments if they process experiences that are more common in these environments. For example, repetitive use of language systems will lead to stronger connections between language-processing regions ${ }^{156,157}$. By contrast, rare experiences should signal that the environment is still changing and that plasticity is beneficial. Gopnik $^{158}$ has argued that humans have extended childhoods to allow there to be a "turbo-powered super sensitive period" to accommodate our unpredictable environments. Computational evolutionary models suggest that children with more 
variable experiences, regardless of the valence of these experiences, reduce their estimate of uncertainty about the environment later, and hence lose plasticity later than do children who experience less-variable environments ${ }^{159-161}$. Environmental variability may also be intrinsically rewarding, increasing dopamine levels, thereby boosting plasticity ${ }^{162-164}$.

We expect the valence and timing of experiences to interact. We suggest a model that predicts that experiences that are both negative and chronic or repeated are the most likely to accelerate brain development and reduce plasticity. Repeated exposure to negative experiences would lead to maturation of the networks that process these experiences, and would augment glucocorticoid levels, allostatic load and inflammatory processes that age the entire body. By contrast, experiences that are positive and rare are predicted by our model to be the most likely to decelerate brain development and enhance plasticity. The hormonal and neurochemical sequelae of positive experiences are not as well studied as those of negative experiences, but awe and surprise have been associated with the release of neurotransmitters associated with enhanced plasticity, including dopamine and acetylcholine ${ }^{165,166}$. Positive social interactions lead to oxytocin release, which has also been shown to enhance plasticity ${ }^{167}$. We expect that experiences that are negative and rare, such as acute traumas, may not necessarily have major impacts on the rate of global maturation, but that specific aspects of those experiences, such as their developmental timing, severity and broader context, may be important in determining their impact on development and plasticity. Similarly, experiences that are positive and repeated may not necessarily broadly impact the rate of global maturation. Indeed, some evidence suggests that in humans cognitive enrichment (or its converse, deprivation) has little effect on the pace of cellular ageing or pubertal timing ${ }^{92}$. Future empirical work will help us refine a model of how specific aspects of early experiences alter the pace of brain development, with consequences for cognition and learning.

\section{Future directions and conclusions}

In this Perspective, we have considered evidence that experiences associated with childhood SES affect not only the outcome but also the pace of brain development, with potential influences on brain plasticity throughout life. We argue that low exposure to stress and high exposure to novel positive experiences promote protracted structural brain development, which gives rise to a later, longer trajectory of functional network segregation, ultimately leading to more efficient cortical networks in adulthood.

However, this model is based on incomplete data. Studies to date have not been fully representative of human diversity, focusing primarily on Western populations with nutritional excess ${ }^{168-170}$. Studies have also been limited by methodological challenges, cross-sectional samples, lack of connection to adult research and correlational designs. Below, we discuss promising approaches to overcome these limitations and directly test our hypotheses in future research.

Methodological advances are necessary to fully understand how early experiences affect the pace of brain development. The application of network methods to developmental data is still in its infancy, as researchers take on the challenge of describing nodes and edges of brain networks in a biologically accurate and meaningful way ${ }^{171,172}$. Studies have used many different measures of segregation to characterize functional brain networks, and it will be crucial for future research to examine how different measures relate to each other and to SES over development. The field has also become increasingly aware of how methodological decisions, including correcting for head motion ${ }^{173-176}$ and physiological artefacts ${ }^{177-179}$, affect study conclusions, and thus affect our ability to make inferences across sets of studies. New methods are also needed for integrating structural and functional brain data. Few articles have examined both functional and structural brain development in the context of SES, and little is known about the relative ordering of trajectories of cortical thinning, white matter development and functional network segregation. Recent work has attempted to link changes in structure to changes in function ${ }^{54,180}$, but the sequence of developmental progression, let alone environmental influences on that sequence, remains murky. Another area for future work involves linking histology and electrophysiology data to structural and functional MRI findings in animal models to facilitate translation to human work. Such an effort would enable us to test how early-life experiences influence cellular developmental processes, including myelination and inhibition, that give rise to macro-level measures, including cortical thickness, surface area and network segregation.

Many of the studies reviewed herein are cross-sectional. Cross-sectional data have inherent limitations when developmental processes are being examined, foremost among them the inability to infer the shape of developmental trajectories ${ }^{9}$. Cross-sectional studies cannot establish temporal precedence and, if sampling is non-random, associations with age may be driven by the characteristics of the sample rather than by age ${ }^{181,182}$. Longitudinal studies, such as the Adolescent Brain Cognitive Development (ABCD) study ${ }^{183,184}$ and the upcoming HEALthy Brain and Child Development (HBCD) study ${ }^{185}$, will be necessary to fully understand how early environments influence trajectories of functional and structural brain development ${ }^{182,186}$. Data from these longitudinal studies will enable us to examine whether changes in brain structure correspond to changes in functional network segregation, and whether measures of the early environment predict earlier or later peaks in these trajectories.

An important future direction is determining whether SES effects on early brain maturation set the stage for early brain ageing ${ }^{3,187,188}$. There is initial evidence from a prospective study that traces of childhood SES are still present in the brain structure of young adults aged 23-25 years, even when adulthood SES is controlled for ${ }^{189}$. We do not yet know whether this is also true of older adults, but studies suggest that cognitive enrichment might be important: cognitive stimulation in childhood is associated with larger brain volumes ${ }^{190}$ and better cognition in old age $^{191}$ when adulthood SES is controlled for. Furthermore, a longitudinal study showed that higher levels of early cognitive stimulation are associated with slower cognitive decline and less neuropathology with ageing ${ }^{192}$. Studies examining adulthood SES and brain structure and function find results that are broadly consistent with the theoretical framework we outline in this Perspective ${ }^{193-196}$. In one study, adults from higher-SES backgrounds showed a weaker negative association between segregation and age than did adults from lower-SES backgrounds, consistent with an interpretation of a slower decline in functional network organization in higher-SES adults ${ }^{197}$. Associations with adulthood SES were stronger than associations with childhood SES; however, because adulthood SES and childhood SES are correlated, these factors can be difficult to disentangle. Childhood SES is difficult to measure in an ageing sample because of recall biases ${ }^{198}$. Relationships between parental education and childhood experiences may also have been different 
when today's 80 -year-olds were children from how they are for today's 6-year-olds, making retrospective report of SES in adults difficult to map to current developmental research. In addition, low-SES populations may be poorly represented in ageing research, owing to lower-life expectancy ${ }^{187}$ and higher prevalence of other diseases and health issues that would exclude these populations from studies of healthy ageing ${ }^{3,188}$. Ideally, future studies will follow individuals from birth to old age, although this may be more feasible in animal models (for example, as in REF. ${ }^{199}$ ).

Although longitudinal observational studies are useful, intervention studies are necessary to directly test whether children's early experiences cause slower or faster brain development. Future work should test whether cognitive enrichment in humans leads to changes in the pace of brain development, and whether the timing of enrichment influences these effects. Although we cannot evaluate the impact of creating early stressful experiences for children, we can learn from the effect of naturally occurring stressors. The emergence of severe acute respiratory syndrome coronavirus 2 (SARS-CoV-2) as a global public health crisis has resulted in an unforeseen natural experiment on how the timing - that is, the age of children when it occurred - and the severity of a stressor affect the pace of children's maturation. However, the effect of the stress has been non-random, as the crisis has disproportionately affected lower-income communities and people from minority racial or ethnic groups and other marginalized populations ${ }^{200,201}$. Following the brain development of children who lived through this period will yield insight into the importance of stress timing on the rate of maturation.

It is possible to investigate causal effects of cognitive enrichment by studying educational interventions. Education is broadly beneficial for children's development, leading to increased cognitive ability, and better health and wellness throughout life ${ }^{202,203}$. Both the type and the timing of education could influence brain plasticity. Rote practice is likely to drive faster maturation of the brain systems involved, which would be beneficial for the task practised (for example, reading and writing), but it could compromise the ability to learn novel tasks. By contrast, rich and varied experiences that capture children's attention and enhance their motivation, boosting levels of acetylcholine and dopamine, could decelerate the rate of brain maturation. Our model also predicts that educational experiences earlier in childhood will have a bigger effect on brain development and plasticity than experiences later in childhood, by changing the trajectory of maturation. Evidence for the efficacy of early interventions, such as from the Abecedarian Project and the Perry Preschool Program, is broadly consistent with this hypothesis ${ }^{204,205}$; however, direct comparisons of the same curricula at different ages are rare, and thus the neural outcomes of changing the timing of such interventions are not yet known. Determining the consequences of educational strategies for the pace of brain maturation is an important area of future research.

In conclusion, disparate strands of evidence from neuroscience, psychology and medicine are consistent with a model in which the early environment affects not only the outcome but also the pace of human brain development. We propose that high stress and low cognitive enrichment accelerate developmental changes in cortical thickness and surface area, and shift the trajectory and amplitude of functional network segregation across development. We argue that changes in the pace of brain development also affect plasticity during development. Our work provides a generative theoretical framework for research on links between childhood experiences and brain changes over the lifespan, and reinforces the pressing need to elucidate changes in early development that lead to disparities in later-life outcomes. If we can develop new screening tools to detect accelerated development, we will be better able to implement educational and other interventions earlier, and prevent cascading consequences of early maturation for mental and physical health.

\section{Citation diversity statement}

Recent work in neuroscience and other fields has identified a bias in citation practices such that articles authored by women and scholars from minority racial or ethnic groups are undercited relative to the number of such articles in the field ${ }^{206-208}$. The expected gender proportions in reference lists of five top neuroscience journals as reported by Dworkin et al. were $6.7 \%$ for woman (first author)/woman (last author), $9.4 \%$ for man/woman, $25.5 \%$ for woman/man and $58.4 \%$ for $\mathrm{man} / \mathrm{man}^{209}$. Inclusion of citation diversity statements has been proposed as a way of increasing transparency surrounding citation practice $^{210,211}$. We obtained the predicted gender of the first and last authors of each reference by using databases that store the probability of a name belonging to a woman or man, and classifying as 'unknown' any names with under $70 \%$ predicted accuracy $^{209,212}$. Excluding self-citations of the authors of the current Perspective, for our references the proportions are $26 \%$ woman (first author)/woman (last author), 20\% man/woman, $21 \%$ woman/man and $33 \%$ $\mathrm{man} / \mathrm{man}$. This method is limited in that the databases used may not, in every case, be indicative of gender identity, and in that it does not account for intersex, non-binary or transgender people. In addition, the expected proportions above were calculated across all neuroscience subfields, and may differ for particular subfields, such as developmental neuroscience. We look forward to future work to better understand how to support equitable practices in science.

Ursula A. Tooley (iD ${ }^{1,2}$, Danielle S. Bassett (iD $3,4,5,6,7,8$ and Allyson P. Mackey iD ${ }^{2 \times}$

${ }^{1}$ Neuroscience Graduate Group, Perelman School of Medicine, University of Pennsylvania, Pennsylvania, $P A, U S A$.

${ }^{2}$ Department of Psychology, School of Arts and Sciences, University of Pennsylvania, Philadelphia, PA, USA.

${ }^{3}$ Department of Bioengineering, School of Engineering and Applied Science, University of Pennsylvania, Philadelphia, PA, USA.

${ }^{4}$ Department of Electrical and Systems Engineering, School of Engineering and Applied Science, University of Pennsylvania, Philadelphia, PA, USA.

${ }^{5}$ Department of Physics \& Astronomy, College of Arts \& Sciences, University of Pennsylvania, Philadelphia, $P A, U S A$.

${ }^{6}$ Department of Neurology, Perelman School of Medicine, University of Pennsylvania, Philadelphia, $P A, U S A$.

${ }^{7}$ Department of Psychiatry, Perelman School of Medicine, University of Pennsylvania, Philadelphia, $P A, U S A$.

${ }^{8}$ Santa Fe Institute, Santa Fe, NM, USA.

凶e-mail:mackeya@upenn.edu

https://doi.org/10.1038/s41583-021-00457-5 Published online 28 April 2021

1. Duncan, G. J., Magnuson, K., Kalil, A. \& Ziol-Guest, K. The importance of early childhood poverty. Soc. Indic. Res. 108, 87-98 (2012).

2. Duncan, G. J., Yeung, W. J., Brooks-Gunn, J. \& Smith, J. R. How much does childhood poverty affect the life chances of children? Am. Sociol. Rev. 63, 406-423 (1998).

3. Cohen, S., Janicki-Deverts, D., Chen, E. \& Matthews, K. A. Childhood socioeconomic status and adult health. Ann. N. Y. Acad. Sci. 1186, 37-55 (2010).

4. Evans, G. W. \& Cassells, R. C. Childhood poverty, cumulative risk exposure, and mental health in emerging adults. Clin. Psychol. Sci. J. Assoc. Psychol. Sci. 2, 287-296 (2014).

5. Farah, M. J. The neuroscience of socioeconomic status: correlates, causes, and consequences. Neuron 96, 56-71 (2017).

6. Hair, N. L., Hanson, J. L., Wolfe, B. L. \& Pollak, S. D. Association of child poverty, brain development, and academic achievement. JAMA Pediatr. 169, 822 (2015). 
7. Finn, A. S. et al. Functional brain organization of working memory in adolescents varies in relation to family income and academic achievement. Dev. Sci. 20, e12450 (2017)

8. Mackey, A. P. et al. Neuroanatomical correlates of the income-achievement gap. Psychol. Sci. 26, 925-933 (2015).

9. Kraemer, H. C., Yesavage, J. A., Taylor, J. L. \& Kupfer, D. How can we learn about developmental processes from cross-sectional studies, or can we? Am. J. Psychiatry 157, 163-171 (2000).

10. Innocenti, G. M. \& Price, D. J. Exuberance in the development of cortical networks. Nat. Rev. Neurosci. 6, 955-965 (2005)

11. Huttenlocher, P. R. $\&$ Dabholkar, A. S. Regional differences in synaptogenesis in human cerebral cortex J. Comp. Neurol 387, 167-178 (1997).

12. Li, G., Lin, W., Gilmore, J. H. ¿ Shen, D. Spatial patterns, longitudinal development, and hemispheric asymmetries of cortical thickness in infants from birth to 2 years of age. J. Neurosci. 35, 9150-9162 (2015).

13. Stiles, J. \& Jernigan, T. L. The basics of brain development. Neuropsychol. Rev. 20, 327-348 (2010).

14. Natu, V. S. et al. Apparent thinning of human visual cortex during childhood is associated with myelination. Proc. Natl Acad. Sci. USA https:// doi.org/10.1073/pnas. 1904931116 (2019).

15. Goriounova, N. A. et al. Large and fast human pyramidal neurons associate with intelligence. eLife 7 e41714 (2018)

16. Brown, T. T. \& Jernigan, T. L. Brain development during the preschool years. Neuropsychol. Rev. 22 , 313-333 (2012)

17. Schnack, H. G. et al. Changes in thickness and surface area of the human cortex and thei relationship with intelligence. Cereb. Cortex 25 1608-1617 (2015)

18. Lawson, G. M., Duda, J. T., Avants, B. B., Wu, J. \& Farah, M. J. Associations between children's socioeconomic status and prefrontal cortical thickness. Dev. Sci. 16, 641-652 (2013).

19. Leonard, J. A. et al. Associations between cortical thickness and reasoning differ by socioeconomic status in development. Dev. Cogn. Neurosci. https:// doi.org/10.1016/j.den.2019.100641 (2019).

20. Alnæs, D., Kaufmann, T., Marquand, A. F. Smith, S. M. \& Westlye, L. T. Patterns of sociocognitive stratification and perinatal risk in the child brain. Proc. Natl Acad. Sci. USA https://doi.org/10.1073/ pnas. 2001517117 (2020).

21. Jha, S. C. et al. Environmental influences on infant cortical thickness and surface area. Cereb. Cortex 29, 1139-1149 (2019)

22. Piccolo, L. R., Merz, E. C., He, X., Sowell, E. R. \& Noble, K. G. Age-related differences in cortical thickness vary by socioeconomic status. PLOS ONE 11 e0162511 (2016).

23. Khundrakpam, B. et al. Non-linear effects of socioeconomic status on brain development associations between parental occupation, cortical thickness and language skills in childhood and adolescence. bioRxiv https://doi.org/10.1101/575993 (2019).

24. Parker, N. et al. Income inequality, gene expression, and brain maturation during adolescence. Sci. Rep. 7 7397 (2017)

25. McDermott, C. L. et al. Longitudinally mapping childhood socioeconomic status associations with cortical and subcortical morphology. J. Neurosci. https://doi.org/10.1523/JNEUROSCl.1808-18.2018 (2018).

26. Judd, N. et al. Cognitive and brain development is independently influenced by socioeconomic status and polygenic scores for educational attainment. Proc. Natl Acad. Sci. USA 117, 12411-12418 (2020).

27. Noble, K. G. et al. Family income, parental education and brain structure in children and adolescents. Nat. Neurosci. 18, 773-778 (2015).

28. LeWinn, K. Z., Sheridan, M. A., Keyes, K. M., Hamilton, A. \& McLaughlin, K. A. Sample composition alters associations between age and brain structure. Nat. Commun. 8, 874 (2017)

29. Chechik, G., Meilijson, I. \& Ruppin, E. Synaptic pruning in development: a novel account in neura terms. in Computational Neuroscience (ed. Bower, J. M.) 149-154 (Springer, 1998).

30. Baxter, R. A. \& Levy, W. B. Constructing multilayered neural networks with sparse, data-driven connectivity using biologically-inspired, complementary, homeostatic mechanisms. Neural Netw. 122, 68-93 (2020).
31. Thomas, M. S. C. Do more intelligent brains retain heightened plasticity for longer in development? A computational investigation. Dev. Cogn. Neurosci. 19, 258-269 (2016)

32. Ju, H., Colbert, C. M. \& Levy, W. B. Limited synapse overproduction can speed development but sometimes with long-term energy and discrimination penalties. PLOS Comput. Biol. 13, e 1005750 (2017).

33. Church, J. A., Petersen, S. E. \& Schlaggar, B. L. The "task B problem" and other considerations in developmental functional neuroimaging. Hum. Brain Mapp. 31, 852-862 (2010).

34. Fox, M. D. \& Raichle, M. E. Spontaneous fluctuations in brain activity observed with functional magnetic resonance imaging. Nat. Rev. Neurosci. 8, 700-71 (2007).

35. Wig, G. S. Segregated systems of human brain networks. Trends Cogn. Sci. 21, 981-996 (2017).

36. Gur, R. E. et al. Burden of environmental adversity associated with psychopathology, maturation, and brain behavior parameters in youths. JAMA Psychiatry https://doi.org/10.1001/jamapsychiatry.2019.0943 (2019).

37. Marshall, N. A. et al. Socioeconomic disadvantage and altered corticostriatal circuitry in urban youth Hum. Brain Mapp. 39, 1982-1994 (2018).

38. Fareri, D. S. et al. Normative development of ventral striatal resting state connectivity in humans. Neurolmage 118, 422-437 (2015)

39. Hanson, J. L. et al. Resting state coupling between the amygdala and ventromedial prefrontal cortex is related to household income in childhood and indexes future psychological vulnerability to stress. Dev. Psychopathol. https://doi.org/10.1017/ S0954579419000592 (2019)

40. Barch, D. et al. Effect of hippocampal and amygdala connectivity on the relationship between preschool poverty and school-age depression. Am. J. Psychiatry 173, 625-634 (2016).

41. Gabard-Durnam, L. J. et al. The development of human amygdala functional connectivity at rest from 4 to 23 years: a cross-sectional study. Neurolmage 95 193-207 (2014)

42. Guerra-Carrillo, B., Mackey, A. P. \& Bunge, S. A Resting-state fMRI: a window into human brain plasticity. Neuroscientist 20, 522-533 (2014).

43. Bassett, D. S. \& Sporns, O. Network neuroscience. Nat. Neurosci. 20, 353-364 (2017).

44. Bassett, D. S., Zurn, P. \& Gold, J. I. On the nature and use of models in network neuroscience. Nat. Rev. Neurosci. 19, 566 (2018)

45. Bassett, D. S., Xia, C. H. \& Satterthwaite, T. D. Understanding the emergence of neuropsychiatric disorders with network neuroscience. Biol. Psychiatry Cogn. Neurosci. Neuroimaging 3, 742-753 (2018).

46. Zuo, X.-N. et al. Human connectomics across the life span. Trends Cogn. Sci. 21, 32-45 (2017).

47. Petersen, S. E. \& Sporns, O. Brain networks and cognitive architectures. Neuron 88, 207-219 (2015).

48. Bassett, D. S. \& Bullmore, E. T. Small-world brain networks revisited. Neuroscientist 23, 499-516 (2017).

49. Gu, S. et al. Emergence of system roles in normative neurodevelopment. Proc. Natl Acad. Sci. USA 112, 13681-13686 (2015).

50. Thomason, M. E. et al. Intrinsic functional brain architecture derived from graph theoretical analysis in the human fetus. PLOS ONE 9, e94423 (2014).

51. Zhao, T., Xu, Y. \& He, Y. Graph theoretical modeling of baby brain networks. Neurolmage https://doi.org 10.1016/i.neuroimage. 2018.06 .038 (2018).

52. Kaiser, M. \& Varier, S. Evolution and development of brain networks: from Caenorhabditis elegans to Homo sapiens. Netw. Comput. Neural Syst. 22, 143-147 (2011).

53. Kaiser, M. Mechanisms of connectome development. Trends Cogn. Sci. 21, 703-717 (2017).

54. Larivière, S. et al. Multiscale structure-function gradients in the neonatal connectome. Cereb. Cortex https://doi.org/10.1093/cercor/bhz069 (2019).

55. Eyre, M. et al. The developing human connectome project: typical and disrupted functional connectivity across the perinatal period. bioRxiv https://doi.org/ 10.1101/2020.01.20.912881 (2020)

56. Grayson, D. S. \& Fair, D. A. Development of large-scale functional networks from birth to adulthood: a guide to the neuroimaging literature. Neurolmage 160 , 15-31 (2017)

57. Marek, S., Hwang, K., Foran, W., Hallquist, M. N. \& Luna, B. The contribution of network organization and integration to the development of cognitive control. PLoS Biol. 13, e1002328 (2015).

58. Kraft, A. W. et al. Electrically coupled inhibitory interneurons constrain long-range connectivity of cortical networks. Neurolmage 215, 116810 (2020).

59. Heuvel, M. P., van den, Scholtens, L. H., Barrett, L. F., Hilgetag, C. C. \& de.Reus, M. A. Bridging cytoarchitectonics and connectomics in human cerebral cortex. J. Neurosci. 35, 13943-13948 (2015).

60. Scholtens, L. H., Schmidt, R., Reus, M. A. de \& van den Heuvel, M. P. Linking macroscale graph analytical organization to microscale neuroarchitectonics in the macaque connectome. J. Neurosci. 34 , 12192-12205 (2014).

61. Wei, Y., Scholtens, L. H., Turk, E. \& van den Heuvel, M. P. Multiscale examination of cytoarchitectonic similarity and human brain connectivity. Netw. Neurosci. https:// doi.org/10.1162/netn_a_00057 (2018).

62. van den Heuvel, M. P. et al. Multimodal analysis of cortical chemoarchitecture and macroscale fMRI resting-state functional connectivity: chemoarchitecture and fMRI connectivity. Hum. Brain Mapp. 37 , 3103-3113 (2016)

63. Gao, W. et al. Functional network development during the first year: relative sequence and socioeconomic correlations. Cereb. Cortex 25, 2919-2928 (2015)

64. Tooley, U. A. et al. Associations between neighborhood SES and functional brain network development. Cereb. Cortex 30, 1-19 (2020).

65. Gellci, K. et al. Community and household-level socioeconomic disadvantage and functional organization of the salience and emotion network in children and adolescents. Neurolmage 184, 729-740 (2019).

66. Noble, K. G., Houston, S. M., Kan, E. \& Sowell, E. R. Neural correlates of socioeconomic status in the developing human brain: neural correlates of socioeconomic status. Dev. Sci. 15, 516-527 (2012).

67. McLaughlin, K. A., Sheridan, M. A. \& Lambert, H. K. Childhood adversity and neural development: Deprivation and threat as distinct dimensions of early experience. Neurosci. Biobehav. Rev. 47, 578-591 (2014).

68. Sheridan, M. A. \& McLaughlin, K. A. Dimensions of early experience and neural development: deprivation and threat. Trends Cogn. Sci. 18 580-585 (2014).

69. McLaughlin, K. A. \& Sheridan, M. A. Beyond cumulative risk: a dimensional approach to childhood adversity. Curr. Dir. Psychol. Sci. 25, 239-245 (2016).

70. Baum, A., Garofalo, J. P. \& Yali, A. M. Socioeconomic status and chronic stress: does stress account for SES effects on health? Ann. N. Y. Acad. Sci. 896, 131-144 (1999).

71. Callaghan, B. L. \& Tottenham, N. The stress acceleration hypothesis: effects of early-life adversity on emotion circuits and behavior. Curr. Opin. Behav. Sci. 7, 76-81 (2016).

72. Callaghan, B. L. \& Tottenham, N. The neuroenvironmental loop of plasticity: a cross-species analysis of parental effects on emotion circuitry development following typical and adverse caregiving Neuropsychopharmacology 41, 163-176 (2016).

73. Monroe, S. M. Modern approaches to conceptualizing and measuring human life stress. Annu. Rev. Clin. Psychol. 4, 33-52 (2008)

74. Belsky, J. Early-life adversity accelerates child and adolescent development. Curr. Dir. Psychol. Sci. 28 241-246 (2019).

75. Short, A. K. \& Baram, T. Z. Early-life adversity and neurological disease: age-old questions and novel answers. Nat. Rev. Neurol. https://doi.org/10.1038/ s41582-019-0246-5 (2019).

76. Gee, D. G. et al. Early developmental emergence of human amygdala-prefrontal connectivity after maternal deprivation. Proc. Natl Acad. Sci. USA 110, 15638-15643 (2013)

77. Herringa, R. J. et al. Enhanced prefrontal-amygdala connectivity following childhood adversity as a protective mechanism against internalizing in adolescence. Biol. Psychiatry Cogn. Neurosci. Neuroimaging 1, 326-334 (2016).

78. McEwen, B. S. Stress, adaptation, and disease. Allostasis and allostatic load. Ann. N. Y. Acad. Sci. 840, 33-44 (1998).

79. Zhang, X., Chen, X. \& Zhang, X. The impact of exposure to air pollution on cognitive performance. Proc. Natl Acad. Sci. USA 115, 9193-9197 (2018). 
80. Mezick, E. J. et al. Influence of race and socioeconomic status on sleep: Pittsburgh sleep SCORE project. Psychosom. Med. 70, 410-416 (2008).

81. Wen, M., Zhang, X., Harris, C. D., Holt, J. B. \& Croft, J. B. Spatial disparities in the distribution of parks and green spaces in the USA. Ann. Behav. Med. Publ. Soc. Behav. Med. 45, 18-27 (2013).

82. Martens, D. S. et al. Prenatal air pollution and newborns' predisposition to accelerated biological aging. JAMA Pediatr. 171, 1160 (2017).

83. James, S. et al. Sleep duration and telomere length in children J Pediatr 187, 247-252 e1 (2017).

84. Irwin, M. R., Olmstead, R. \& Carroll, J. E. Sleep disturbance, sleep duration, and inflammation: a systematic review and meta-analysis of cohort studies and experimental sleep deprivation. Biol. Psychiatry 80, 40-52 (2016).

85. Miller, G. E., Chen, E. \& Parker, K. J. Psychological stress in childhood and susceptibility to the chronic diseases of aging: moving toward a model of behavioral and biological mechanisms. Psychol. Bull. 137, 959-997 (2011).

86. Lam, L. L. et al. Factors underlying variable DNA methylation in a human community cohort. Proc. Natl Acad. Sci. USA 109, 17253-17260 (2012).

87. Mitchell, C. et al. Social disadvantage, genetic sensitivity, and children's telomere length. Proc. Natl Acad. Sci. USA 111, 5944-5949 (2014).

88. Austin, M. K. et al. Early-life socioeconomic disadvantage, not current, predicts accelerated epigenetic aging of monocytes. Psychoneuroendocrinology 97, 131-134 (2018).

89. James-Todd, T., Tehranifar, P., Rich-Edwards, J., Titievsky, L. \& Terry, M. B. The impact of socioeconomic status across early life on age at menarche among a racially diverse population of girls. Ann. Epidemiol. 20, 836-842 (2010).

90. Sun, Y., Mensah, F. K., Azzopardi, P., Patton, G. C. \& Wake, M. Childhood social disadvantage and pubertal timing: a national birth cohort from Australia. Pediatrics https://doi.org/10.1542/peds.2016-4099 (2017).

91. Belsky, J., Ruttle, P. L., Boyce, W. T., Armstrong, J. M. \& Essex, M. J. Early adversity, elevated stress physiology, accelerated sexual maturation, and poor health in females. Dev. Psychol. 51, 816-822 (2015).

92. Colich, N. L., Rosen, M. L., Williams, E. S. \& McLaughlin, K. A. Biological aging in childhood and adolescence following experiences of threat and deprivation: a systematic review and meta-analysis. Psychol. Bull. 146, 721-764 (2020).

93. Snell-Rood, E. \& Snell-Rood, C. The developmental support hypothesis: adaptive plasticity in neural development in response to cues of social support. Philos. Trans. R. Soc. B Biol. Sci. 375, 20190491 (2020).

94. Weisbecker, V. \& Goswami, A. Brain size, life history, and metabolism at the marsupial/placental dichotomy. Proc. Natl Acad. Sci. USA 107, 16216-16221 (2010).

95. Snell-Rood, E. C., Davidowitz, G. \& Papaj, D. R. Reproductive tradeoffs of learning in a butterfly. Behav. Ecol. 22, 291-302 (2011).

96. Walker, C.-D. et al. Chronic early life stress induced by limited bedding and nesting (LBN) material in rodents: critical considerations of methodology, outcomes and translational potential. Stress 20 421-448 (2017)

97. Rice, C. J., Sandman, C. A., Lenjavi, M. R. \& Baram, T. Z. A novel mouse model for acute and long-lasting consequences of early life stress. Endocrinology 149, 4892-4900 (2008).

98. Brunson, K. L. et al. Mechanisms of late-onset cognitive decline after early-life stress. J. Neurosci. 25, 9328-9338 (2005)

99. Bath, K. G., Manzano-Nieves, G. \& Goodwill, H. Early life stress accelerates behavioral and neura maturation of the hippocampus in male mice. Horm. Behav. 82, 64-71 (2016).

100. Manzano Nieves, G., Bravo, M., Baskoylu, S. \& Bath, K. G. Early life adversity decreases pre-adolescent fear expression by accelerating amygdala PV cell development. eLife 9, e55263 (2020)

101. Naninck, E. F. G. et al. Chronic early life stress alters developmental and adult neurogenesis and impairs cognitive function in mice. Hippocampus $\mathbf{2 5}$, 309-328 (2015)

102. Ivy, A. S. et al. Hippocampal dysfunction and cognitive impairments provoked by chronic early-life stress involve excessive activation of $\mathrm{CRH}$ receptors. J. Neurosci. 30, 13005-13015 (2010).
103. Yang, X.-D. et al. Stress during a critical postnatal period induces region-specific structural abnormalities and dysfunction of the prefrontal cortex via CRF1. Neuropsychopharmacology 40, 1203-1215 (2015).

104. Danese, A. \& McEwen, B. S. Adverse childhood experiences, allostasis, allostatic load, and age-related disease. Physiol. Behav. 106, 29-39 (2012).

105. Bradley, R. H., Corwyn, R. F., McAdoo, H. P. \& Coll, C. G The home environments of children in the United States part I: variations by age, ethnicity, and poverty status. Child. Dev. 72, 1844-1867 (2001).

106. Sheridan, M. A., Peverill, M., Finn, A. S. \& Mclaughlin, K. A. Dimensions of childhood adversity have distinct associations with neural systems underlying executive functioning. Dev. Psychopathol. 29, 1777-1794 (2017).

107. Rosen, M. L., Sheridan, M. A., Sambrook, K. A., Meltzoff, A. N. \& McLaughlin, K. A. Socioeconomic disparities in academic achievement: a multi-modal investigation of neural mechanisms in children and adolescents. Neurolmage 173, 298-310 (2018).

108. Amso, D., Salhi, C. \& Badre, D. The relationship between cognitive enrichment and cognitive control: a systematic investigation of environmental influences on development through socioeconomic status. Dev Psychobiol 61, 159-178 (2019).

109. Rosen, M. L. et al. Cognitive stimulation as a mechanism linking socioeconomic status with executive function: a longitudinal investigation. Child Dev. https://doi.org/10.1111/cdev.13315 (2019).

110. Diamond, M. C. Response of the brain to enrichment An. Acad. Bras. Ciênc. 73, 211-220 (2001).

111. Bennett, E. L., Diamond, M. C., Krech, D. \& Rosenzweig, M. R. Chemical and anatomical plasticity of brain. Science 146, 610-619 (1964).

112. Diamond, M. C., Krech, D. \& Rosenzweig, M. R The effects of an enriched environment on the histology of the rat cerebral cortex. J. Comp. Neurol. 123, 111-119 (1964).

113. Connor, J. R., Wang, E. C. \& Diamond, M. C. Increased length of terminal dendritic segments in old adult rats somatosensory cortex: an environmentally induced response. Exp. Neurol. 78, 466-470 (1982).

114. Schapiro, S. \& Vukovich, K. R. Early experience effects upon cortical dendrites: a proposed model for development. Science 167, 292-294 (1970).

115. Diamond, M. C. et al. Increases in cortical depth and glia numbers in rats subjected to enriched environment. J. Comp. Neurol. 128, 117-125 (1966).

116. Leal-Galicia, P., Castañeda-Bueno, M., Quiroz-Baez, R. $\&$ Arias, C. Long-term exposure to environmental enrichment since youth prevents recognition memory decline and increases synaptic plasticity markers in aging. Neurobiol. Learn. Mem. 90, 511-518 (2008).

117. Markham, J. A. \& Greenough, W. T. Experience-driven brain plasticity: beyond the synapse. Neuron Glia Biol. 1, 351-363 (2005)

118. Diamond, M. C. Enriching Heredity: The Impact of the Environment on the Anatomy of the Brain (Free Press, 1988)

119. Scholz, J., Allemang-Grand, R., Dazai, J. \& Lerch, J. P. Environmental enrichment is associated with rapid volumetric brain changes in adult mice. Neurolmage 109, 190-198 (2015).

120. Bennett, E. L., Rosenzweig, M., Diamond, M Morimoto, H. \& Hebert, M. Effects of successive environments on brain measures. Physiol. Behav. 12, 621-631 (1974)

121. Petersen, A. Brain Maturation and Cognitive Development: Comparative and Cross-Cultural Perspectives. (Routledge, 2017).

122. Chechik, G., Meilijson, I. \& Ruppin, E. Synaptic pruning in development: a computational accoun Neural Comput. 10, 1759-1777 (1998).

123. Reh, R. K. et al. Critical period regulation across multiple timescales. Proc. Natl Acad. Sci. USA https:// doi.org/10.1073/pnas. 1820836117 (2020).

124. Werker, J. F. \& Hensch, T. K. Critical periods in speech perception: new directions. Annu. Rev. Psychol. 66, 173-196 (2015)

125. Guadagno, A., Verlezza, S., Long, H., Wong, T. P. \& Walker, C.-D. It is all in the right amygdala: increased synaptic plasticity and perineuronal nets in male, but not female, juvenile rat pups after exposure to early-life stress. J. Neurosci. 40, 8276-8291 (2020).

126. Makinodan, M., Rosen, K. M., Ito, S. \& Corfas, G A critical period for social experience-dependent oligodendrocyte maturation and myelination. Science 337 , 1357-1360 (2012).
127. Sumner, J. A., Colich, N. L., Uddin, M., Armstrong, D. $\&$ McLaughlin, K. A. Early experiences of threat, but not deprivation, are associated with accelerated biological aging in children and adolescents. Biol. Psychiatry https://doi.org/10.1016/j. biopsych.2018.09.008 (2018).

128. Piekarski, D. J., Boivin, J. R. \& Wilbrecht, L. Ovarian hormones organize the maturation of inhibitory neurotransmission in the frontal cortex at puberty onset in female mice. Curr. Biol. 27, 1735-1745.e3 (2017).

129. Tanti, A. et al. Child abuse associates with an imbalance of oligodendrocyte-lineage cells in ventromedial prefrontal white matter. Mol. Psychiatry https://doi.org/10.1038/mp.2017.231 (2017).

130. Chao, L. L., Tosun, D., Woodward, S. H., Kaufer, D. $\&$ Neylan, T. C. Preliminary evidence of increased hippocampal myelin content in veterans with posttraumatic stress disorder. Front. Behav. Neurosci. 9, 333 (2015)

131. Favuzzi, E. et al. Activity-dependent gating of parvalbumin interneuron function by the perineuronal net protein brevican. Neuron 95, 639-655.e10 (2017).

132. Duffy, S. N. Environmental enrichment modifies the PKA-dependence of hippocampal LTP and improves hippocampus-dependent memory Learn. Mem. 8 , 26-34 (2001)

133. O'Connor, A. M. et al. Environmental enrichment from birth impacts parvalbumin expressing cells and wisteria floribunda agglutinin labelled peri-neuronal nets within the developing murine striatum. Front. Neuroanat. 13, 90 (2019)

134. Carstens, K. E., Phillips, M. L., Pozzo-Miller, L., Weinberg, R. J. \& Dudek, S. M. Perineuronal nets suppress plasticity of excitatory synapses on CA2 pyramidal neurons. J. Neurosci. 36, 6312-6320 (2016).

135. Brainard, M. S. \& Knudsen, E. I. Sensitive periods for visual calibration of the auditory space map in the barn owl optic tectum. J. Neurosci. 18, 3929-3942 (1998).

136. Greifzu, F. et al. Environmental enrichment extends ocular dominance plasticity into adulthood and protects from stroke-induced impairments of plasticity. Proc. Natl Acad. Sci. USA 111, 1150-1155 (2014).

137. Greifzu, F., Kalogeraki, E. \& Löwel, S. Environmental enrichment preserved lifelong ocular dominance plasticity, but did not improve visual abilities Neurobiol. Aging 41, 130-137 (2016)

138. Baroncelli, L. et al. Nurturing brain plasticity: Impact of environmental enrichment. Cell Death Differ. 17 1092-1103 (2010)

139. Sale, A. et al. Environmental enrichment in adulthood promotes amblyopia recovery through a reduction of intracortical inhibition. Nat. Neurosci. 10, 679-681 (2007).

140. Foscarin, S. et al. Experience-dependent plasticity and modulation of growth regulatory molecules at central synapses. PLoS ONE 6, e16666 (2011).

141. Nguyen, P. T. et al. Microglial remodeling of the extracellular matrix promotes synapse plasticity. Cell 182, 388-403.e15 (2020)

142. Hughes, E. G., Orthmann-Murphy, J. L., Langseth, A. J. \& Bergles, D. E. Myelin remodeling through experience-dependent oligodendrogenesis in the adult somatosensory cortex. Nat. Neurosci. 21, 696-706 (2018).

143. Takesian, A. E., Bogart, L. J., Lichtman, J. W. \& Hensch, T. K. Inhibitory circuit gating of auditory critical-period plasticity. Nat. Neurosci. 21, 218-227 (2018).

144. Bao, S., Chan, V. T. \& Merzenich, M. M. Cortical remodelling induced by activity of ventral tegmental dopamine neurons. Nature 41 2, 79-83 (2001).

145. Vetencourt, J. F. M. et al. The antidepressant fluoxetine restores plasticity in the adult visual cortex. Science 320, 385-388 (2008).

146. Morishita, H., Miwa, J. M., Heintz, N. \& Hensch, T. K. Plasticity in adult visual cortex. Science 330, 4 (2010).

147. Thanos, P. K. et al. Dopamine D2 gene expression interacts with environmental enrichment to impact lifespan and behavior. Oncotarget 7, 19111-19123 (2016).

148. Grady, D. L. et al. DRD4 genotype predicts longevity in mouse and human. J. Neurosci. 33, 286-291 (2013).

149. Pekarek, B. T., Hunt, P. J. \& Arenkiel, B. R. Oxytocin and sensory network plasticity. Front. Neurosci. 14 $30(2020)$ 
150. Park, S.-H., Kim, Y.-J., Park, J.-C., Han, J.-S. \& Choi, S.-Y. Intranasal oxytocin following uncontrollable stress blocks impairments in hippocampal plasticity and recognition memory in stressed rats. Int. $J$. Neuropsychopharmacol. 20, 861-866 (2017)

151. Crane, J. W., Holmes, N. M., Fam, J., Westbrook, R. F. \& Delaney, A. J. Oxytocin increases inhibitory synaptic transmission and blocks development of long-term potentiation in the lateral amygdala. J. Neurophysiol. 123, 587-599 (2019).

152. Bassett, D. S., Yang, M., Wymbs, N. F. \& Grafton, S. T Learning-induced autonomy of sensorimotor systems. Nat. Neurosci. 18, 744-751 (2015).

153. Finc, K. et al. Dynamic reconfiguration of functional brain networks during working memory training Nat. Commun. 11, 2435 (2020).

154. Mohr. H. et al. Integration and segregation of large-scale brain networks during short-term task automatization. Nat. Commun. 7, 13217 (2016)

155. Rueda, M. R., Rothbart, M. K., McCandliss, B. D. Saccomanno, L. \& Posner, M. I. Training, maturation, and genetic influences on the development of executive attention. Proc. Natl Acad. Sci. USA 102, 14931-14936 (2005).

156. Romeo, R. R. et al. Beyond the " 30 million word gap:" children's conversational exposure is associated with language-related brain function. Psychol. Sci. 29, 700-710 (2018)

157. Romeo, R. R. et al. Language exposure relates to structural neural connectivity in childhood. J. Neurosci. 38, 7870-7877 (2018)

158. Gopnik, A. Childhood as a solution to explore-exploit tensions. Philos. Trans. R. Soc. B Biol. Sci. 375 , 20190502 (2020).

159. Frankenhuis, W. E. \& Panchanathan, K. Individual differences in developmental plasticity may result from stochastic sampling. Perspect. Psychol. Sci. 6, 336-347 (2011)

160. Frankenhuis, W. E. \& Walasek, N. Modeling the evolution of sensitive periods. Dev. Cogn. Neurosci. 41, 100715 (2020).

161. Panchanathan, K. \& Frankenhuis, W. E. The evolution of sensitive periods in a model of incremental development. Proc. R. Soc. B Biol. Sci. 283, 20152439 (2016).

162. Zhu, J., Apparsundaram, S., Bardo, M. T. $\delta$ Dwoskin, L. P. Environmental enrichment decreases cell surface expression of the dopamine transporter in rat medial prefrontal cortex. J. Neurochem. 93 , 1434-1443 (2005)

163. Kim, M.-S. et al. Environmental enrichment enhances synaptic plasticity by internalization of striatal dopamine transporters. J. Cereb. Blood Flow. Metab. 36, 2122-2133 (2016)

164. Heller, A. S. et al. Association between real-world experiential diversity and positive affect relates to hippocampal-striatal functional connectivity. Nat. Neurosci. https://doi.org/10.1038/s41593-0200636-4 (2020)

165. Schomaker, J. \& Meeter, M. Short- and long-lasting consequences of novelty, deviance and surprise on brain and cognition. Neurosci. Biobehav. Rev. $\mathbf{5 5}$ 268-279 (2015)

166. Valdesolo, P., Shtulman, A. \& Baron, A. S. Science is awe-some: the emotional antecedents of science learning. Emot. Rev. 9, 215-221 (2017).

167. Meyer-Lindenberg, A., Domes, G., Kirsch, P. \& Heinrichs, M. Oxytocin and vasopressin in the human brain: social neuropeptides for translational medicine. Nat. Rev. Neurosci. 12, 524-538 (2011).

168. Henrich, J., Heine, S. J. \& Norenzayan, A. The weirdest people in the world? Behav. Brain Sci. 33, 61-83 (2010).

169. Sear, R., Sheppard, P. \& Coall, D. A. Cross-cultural evidence does not support universal acceleration of puberty in father-absent households. Philos. Trans. $R$. Soc. B Biol. Sci. 374, 20180124 (2019).

170. Kyweluk, M. A., Georgiev, A. V., Borja, J. B., Gettler, L. T. \& Kuzawa, C. W. Menarcheal timing is accelerated by favorable nutrition but unrelated to developmental cues of mortality or familial instability in Cebu, Philippines. Evol. Hum. Behav. 39, 76-81 (2018).

171. Wig, G. S., Schlaggar, B. L. \& Petersen, S. E. Concepts and principles in the analysis of brain networks: Brain networks. Ann. N. Y. Acad. Sci. 1224, 126-146 (2011)

172. Bertolero, M. A. \& Bassett, D. S. On the nature of explanations offered by network science: a perspective from and for practicing neuroscientists. Top. Cogn. Sci. https://arxiv.org/abs/1911.05031 (2020).
173. Ciric, R. et al. Mitigating head motion artifact in functional connectivity MRI. Nat. Protoc. https:// doi.org/10.1038/s41596-018-0065-y (2018).

174. Satterthwaite, T. D. et al. Impact of in-scanner head motion on multiple measures of functional connectivity: relevance for studies of neurodevelopment in youth. Neurolmage 60, 623-632 (2012).

175. Parkes, L., Fulcher, B., Yücel, M. \& Fornito, A An evaluation of the efficacy, reliability, and sensitivity of motion correction strategies for resting-state functional MRI. Neurolmage 171, 415-436 (2018).

176. Savalia, N. K. et al. Motion-related artifacts in structural brain images revealed with independent estimates of in-scanner head motion. Hum. Brain Mapp. 38, 472-492 (2017).

177. Fair, D. A. et al. Correction of respiratory artifacts in MRI head motion estimates. Neurolmage $\mathbf{2 0 8}$, 116400 (2020).

178. Power, J. D. et al. Distinctions among real and apparent respiratory motions in human fMRI data. Neurolmage 201, 116041 (2019).

179. Power, J. D. et al. Characteristics of respiratory measures in young adults scanned at rest, including systematic changes and "missed" deep breaths. Neurolmage https://doi.org/10.1016/j.neuroimage. 2019.116234 (2019)

180. Baum, G. L. et al. Development of structure-function coupling in human brain networks during youth. Proc. Natl Acad. Sci.USA 117, 771-778 (2020).

181. Hofer, S. M., Sliwinski, M. J. \& Flaherty, B. P. Understanding ageing: further commentary on the limitations of cross-sectional designs for ageing research. Gerontology 48, 22 (2002)

182. King, K. M. et al. Longitudinal modeling in developmental neuroimaging research: common challenges, and solutions from developmental psychology. Dev. Cogn. Neurosci. 33, 54-72 (2018).

183. Volkow, N. D. et al. The conception of the ABCD study: From substance use to a broad NIH collaboration. Dev. Cogn. Neurosci. 32, 4-7 (2018).

184. Karlsson, L. et al. Cohort profile: the FINNBRAIN birth cohort study (FINNBRAIN). Int. J. Epidemiol. 47 15-16j (2018)

185. Volkow, N. D., Gordon, J. A. \& Freund, M. P. The healthy brain and child development study shedding light on opioid exposure, COVID-19, and health disparities. JAMA Psychiatry https://doi.org/ 10.1001/jamapsychiatry.2020.3803 (2020)

186. Madhyastha, T. et al. Current methods and limitations for longitudinal fMRI analysis across development. Dev. Cogn. Neurosci. 33, 118-128 (2018).

187. Bor, J., Cohen, G. H. \& Galea, S. Population health in an era of rising income inequality: USA, 1980-2015. Lancet 389, 1475-1490 (2017).

188. Braveman, P. A., Cubbin, C., Egerter, S., Williams, D. R. $\&$ Pamuk, E. Socioeconomic disparities in health in the United States: what the patterns tell us. Am. J. Public Health 100, S186-S196 (2010).

189. Dufford, A. J., Evans, G. W., Liberzon, I., Swain, J. E. \& Kim, P. Childhood socioeconomic status is prospectively associated with surface morphometry in adulthood. Dev. Psychobiol. https://doi.org/10.1002/dev.22096 (2020).

190. Moored, K. D. et al. Engagement in enriching early life activities is associated with larger hippocampal and amygdala volumes in community-dwelling older adults. J. Gerontol. Ser. B https://doi.org/10.1093/geronb/ gby 150 (2018)

191. Chan, T., Parisi, J. M., Moored, K. D. \& Carlson, M. C. Variety of enriching early-life activities linked to late-life cognitive functioning in urban community-dwelling African Americans. J. Gerontol. Ser. B 74, 1345-1355 (2019).

192. Oveisgharan, S., Wilson, R. S., Yu, L., Schneider, J. A. $\&$ Bennett, D. A. Association of early-life cognitive enrichment with Alzheimer disease pathological changes and cognitive decline. JAMA Neurol. https://doi.org/10.1001/jamaneurol.2020.1941 (2020).

193. Gianaros, P. J. et al. Community socioeconomic disadvantage in midlife relates to cortical morphology via neuroendocrine and cardiometabolic pathways. Cereb. Cortex 27, 460-473 (2017)

194. Kim, J. P. et al. Effects of education on aging-related cortical thinning among cognitively normal individuals. Neurology 85, 806-812 (2015).

195. Krishnadas, R. et al. Socioeconomic deprivation and cortical morphology: psychological, social, and biological determinants of ill health study. Psychosom. Med. 75, 616-623 (2013).
196. Sripada, R. K., Swain, J. E., Evans, G. W., Welsh, R. C. $\&$ Liberzon, I. Childhood poverty and stress reactivity are associated with aberrant functional connectivity in default mode network. Neuropsychopharmacology 39, 2244-2251 (2014).

197. Chan, M. Y. et al. Socioeconomic status moderates age-related differences in the brain's functional network organization and anatomy across the adult lifespan. Proc. Natl Acad. Sci. USA 115, E5144-E5153 (2018).

198. Baldwin, J. R., Reuben, A., Newbury, J. B. \& Danese, A Agreement between prospective and retrospective measures of childhood maltreatment. JAMA Psychiatry 76, 584-593 (2019)

199. Tung, J., Archie, E. A., Altmann, J. \& Alberts, S. C. Cumulative early life adversity predicts longevity in wild baboons. Nat. Commun. 7, 1-7 (2016).

200. Millett, G. A. et al. Assessing differential impacts of COVID-19 on black communities. Ann. Epidemiol. 47 37-44 (2020).

201. Goyal, M. K. et al. Racial and/or ethnic and socioeconomic disparities of SARS-CoV-2 infection among children. Pediatrics https://doi.org/10.1542/ peds. 2020-009951 (2020)

202. Ritchie, S. J. \& Tucker-Drob, E. M. How much does education improve intelligence? A meta-analysis. Psychol. Sci. 29, 1358-1369 (2018).

203. Adler, N. E. et al. Socioeconomic status and health: the challenge of the gradient. Am. Psychol. 49 15-24 (1994).

204. Heckman, J. J., Moon, S. H., Pinto, R., Savelyev, P. A. $\&$ Yavitz, A. The rate of return to the high/scope perry preschool program. J. Public. Econ. 94, 114-128 (2010).

205. Campbell, F. A., Ramey, C. T., Pungello, E., Sparling, J. \& Miller-Johnson, S. Early childhood education: young adult outcomes from the Abecedarian project. Appl. Dev. Sci. 6, 42-57 (2002).

206. Maliniak, D., Powers, R. \& Walter, B. F. The gender citation gap in international relations. Int. Organ. 67, 889-922 (2013).

207. Caplar, N., Tacchella, S. \& Birrer, S. Quantitative evaluation of gender bias in astronomical publications from citation counts. Nat. Astron. 1, 1-5 (2017).

208. Dion, M. L., Sumner, J. L. \& Mitchell, S. M. Gendered citation patterns across political science and social science methodology fields. Polit. Anal. 26, 312-327 (2018).

209. Dworkin, J. D. et al. The extent and drivers of gender imbalance in neuroscience reference lists. Nat. Neurosci. 23, 918-926 (2020).

210. Dworkin, J., Zurn, P. \& Bassett, D. S. (In)citing action to realize an equitable future. Neuron 106, 890-894 (2020).

211. Zurn, P., Bassett, D. S. \& Rust, N. C. The citation diversity statement: a practice of transparency, a way of life. Trends Cogn. Sci. 24, 669-672 (2020).

212. Zhou, D. et al. Gender Diversity Statement and Code Notebook v1.O. (Zenodo, 2020).

213. Maier-Hein, K. H. et al. The challenge of mapping the human connectome based on diffusion tractography. Nat. Commun. 8, 1-13 (2017)

214. Krogsrud, S. K. et al. Changes in white matter microstructure in the developing brain - a longitudinal diffusion tensor imaging study of children from 4 to 11 years of age. Neurolmage 124, 473-486 (2016).

215. Gilmore, J. H., Knickmeyer, R. C. \& Gao, W. Imaging structural and functional brain development in early childhood. Nat. Rev. Neurosci. 19, 123 (2018).

216. Ozernov-Palchik, O. et al. The relationship between socioeconomic status and white matter microstructure in pre-reading children: A longitudinal investigation. Hum. Brain Mapp. 40, 741-754 (2019).

217. Dufford A \& Kim, P. Family income, cumulative risk exposure, and white matter structure in middle childhood. Front. Hum. Neurosci. 11, 547 (2017).

218. Noble, K. G., Korgaonkar, M. S., Grieve, S. M. $\&$ Brickman, A. M. Higher education is an ageindependent predictor of white matter integrity and cognitive control in late adolescence. Dev. Sci. 16, 653-664 (2013)

219. Takeuchi, H. et al. The effects of family socioeconomic status on psychological and neural mechanisms as well as their sex differences. Front. Hum. Neurosci. 12 543 (2018).

220. Kim, D.-J. et al. Childhood poverty and the organization of structural brain connectome. Neurolmage 184, 409-416 (2019). 


\section{PERSPECTIVES}

221. Hensch, T. K. Critical period plasticity in local cortical circuits. Nat. Rev. Neurosci. 6, 877-888 (2005).

222. Takesian, A. E. \& Hensch, T. K. Balancing plasticity/ stability across brain development. in Progress in Brain Research Vol. 207 3-34 (Elsevier, 2013).

223. Miller, D. J. et al. Prolonged myelination in human neocortical evolution. Proc. Natl Acad. Sci. USA 109 16480-16485 (2012).

224. Glasser, M. F., Goyal, M. S., Preuss, T. M., Raichle, M. E. \& Van Essen, D. C. Trends and properties of human cerebral cortex: correlations with cortical myelin content. Neurolmage 93, 165-175 (2014).

225. Mauney, S. A. et al. Developmental pattern of perineuronal nets in the human prefrontal cortex and their deficit in schizophrenia. Biol. Psychiatry 74 427-435 (2013)

226. Rogers, S. L., Rankin-Gee, E., Risbud, R. M., Porter, B. E. \& Marsh, E. D. Normal development of the perineuronal net in humans; in patients with and without epilepsy. Neuroscience 384, 350-360 (2018).

227. Paredes, M. F. et al. Extensive migration of young neurons into the infant human frontal lobe. Science 354, aaf7073 (2016)

228. Metherate, R. Nicotinic acetylcholine receptors in sensory cortex. Learn. Mem. 11, 50-59 (2004).

\section{Acknowledgements}

The authors thank C. Recto for her assistance with figures, and M. Farah, J. Leonard, Y. Hahn and S. Sharp for their helpful comments on earlier versions of the manuscript. U.A.T. was supported by the US National Science Foundation Graduate Research Fellowship. A.P.M. was supported by a Jacobs Foundation Early Career Research Fellowship and the US National Institute on Drug Abuse (1R34DA050297-01). D.S.B. was supported by the John D. and Catherine T. MacArthur Foundation and the Institute for Scientific Interchange Foundation.
Author contributions

U.A.T. and A.P.M. researched data for the article and contributed substantially to discussion of the content. All authors wrote the article and reviewed and/or edited the manuscript before submission.

\section{Competing interests}

The authors declare no competing interests.

Peer review information

Nature Reviews Neuroscience thanks K. McLaughlin, E. Sowell and the other anonymous, reviewer(s) for their contribution to the peer review of this work.

\section{Publisher's note}

Springer Nature remains neutral with regard to jurisdictional claims in published maps and institutional affiliations.

(c) Springer Nature Limited 2021 